Comm. in Asteroseismology

Vol. 153, 2008

\title{
The new liaison CoAst - HELAS
}

\author{
C. Aerts ${ }^{1}$ for the HELAS Board ${ }^{2}$ \\ ${ }^{1}$ Instituut voor Sterrenkunde, Celestijnenlaan 200D, B-3001 Leuven, Belgium \\ ${ }^{2}$ The HELAS Project Office, Kiepenheuer-Institut für Sonnenphysik, Schöneckstr. 6, \\ D-79104 Freiburg, Germany
}

\begin{abstract}
We report a new agreement between the journal Communications in Asteroseismology and the European Coordination Action in Helio- and Asteroseismology (HELAS).
\end{abstract}

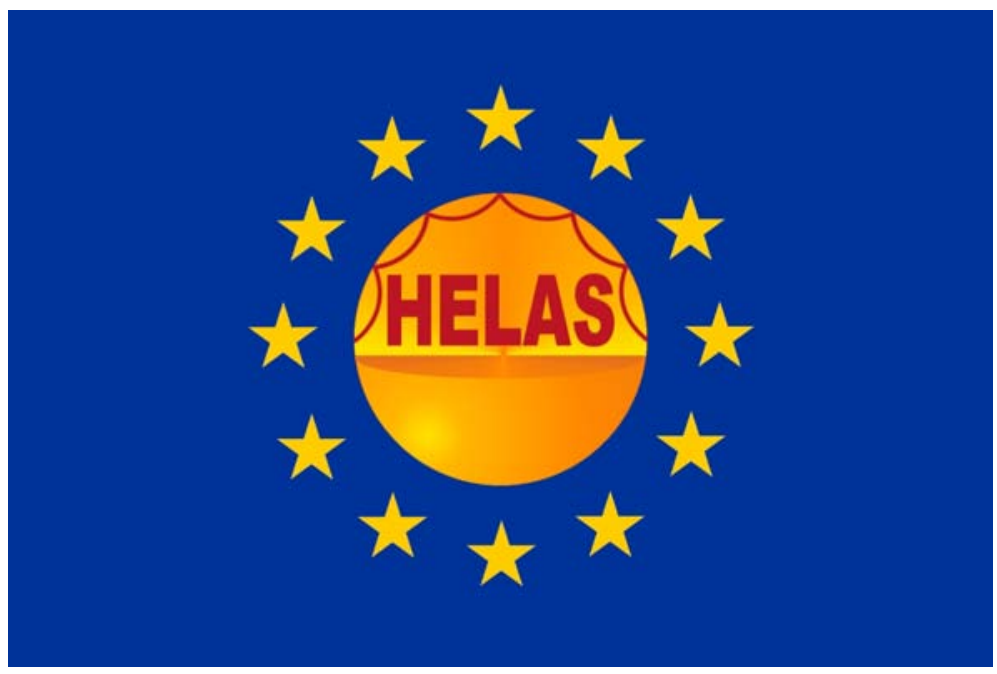

Figure 1: The official logo of HELAS 
HELAS is a Coordination Action funded under the European Commission's Sixth Framework Programme (FP6). Three of its Network Activities concern the coordination of activities in the scientific topics of Global Helioseismology, Local Helioseismology and Asteroseismology across European institutes. Two other activities, Forum and Public Outreach, concentrate on the dissemination of science and knowledge in the three mentioned research fields. Information on the network activities, on meetings organised and supported by HELAS, and on many other topics is available online via http://www.helas-eu.org. We strongly encourage anyone interested in the research fields of HELAS to register through this website.

The Board of HELAS approved the request of the Editor-in-Chief of CoAst, Professor Michel Breger, to provide English language editing for the journal. The Board was of the opinion that this journal is of importance for the dissemination of scientific results in asteroseismology and that the language editing task fits well within the goals of the network. The language editing will be done at the Institute of Astronomy of the University Leuven, which is the HELAS node responsible for the Asteroseismology network activity. The language editing is funded for the remaining two years of the HELAS network.

Given that HELAS already has a monthly electronic newsletter with announcements of jobs, meetings, papers, etc. (to receive it, register at http://www.helas-eu.org), the Board of HELAS decided to fill the HELAS section in CoAst with a thorough description of its five main network activities, as well as with short announcements of deliverables which are considered of value for the whole community (see the example in this issue: the release of the Wroctaw website and the public release of Polish tools for asteroseismology). As a start, we describe in this issue of CoAst the Forum of HELAS.

We welcome any suggestions or comments from the helio- and asteroseismology community, as well as requests for support of initiatives and meetings.

Acknowledgments. This work was supported by the European Helio- and Asteroseismology Network (HELAS), a major international collaboration funded by the European Commission's Sixth Framework Programme. 